\title{
Efeito do tempo, substrato e temperatura na penetração de juvenis do segundo estádio de Meloidogyne javanica e Heterodera glycines em soja
}

\author{
Hercules Diniz Campos ${ }^{1}$, Vicente Paulo Campos², Edson Ampélio Pozza ${ }^{2}$
}

\begin{abstract}
${ }^{1}$ Universidade de Rio Verde -FESURV, Faculdade de Agronomia, CP 104, 75910-970, Rio Verde, GO <campos@ fesurv.br>; 2 Departamento de Fitopatologia, Universidade Federal de Lavras -UFLA, CP 37, 37200-000, Lavras, MG.
\end{abstract}

Data de chegada: 06/05/2004. Aceito para publicação em: 08/09/2005.

\begin{abstract}
Campos, H. D.; Campos, V.P.; Pozza, E.A. Effect of timing, substract and temperature on the penetration of second stage juveniles of Meloidogyne javanica and Heterodera glycines in soybean roots. Summa Phytopathologica, v.32, n. 2, p.156-160, 2006.

At 2 days after inoculation, independent of the substrate used, lower penetration of $\mathrm{J} 2$ of Meloidogyne javanica was observed compared to the other time points tested. When fine sand was used, greater penetration of $\mathrm{J} 2$ of $M$. javanica in soybean roots occurred at 4,4 days after inoculation. By using a mixture of soil and ground sand, the penetration increase was linear between at 2 and 8 days after inoculation. When the fine sand was used number of $\mathrm{J} 2$ of Heterodera glycines, in the roots was highest at 2 days after inoculation. In the mixture of soil and ground sand, the soybean roots had lowest number of $\mathrm{J} 2$ of $\mathrm{H}$. glycines at 2 days after inoculation, decreasing evenly toward day 8 . At $24^{\circ} \mathrm{C}$ the penetration of $\mathrm{J} 2$ of $M$. javanica was higher $(\mathrm{P} \leq 0,05)$ regardless of cultivar resistance. However, in the

temperatures between 24 and $28^{\circ} \mathrm{C}$. At $32^{\circ} \mathrm{C}$ occurred significant decrease in penetration which reached $17,97 \%$ of the penetration observed at $28^{\circ} \mathrm{C}$ and with similar values at 16 and $20^{\circ} \mathrm{C}$. In the susceptible cultivar the penetration decrease was significant at $28^{\circ} \mathrm{C}$ and $32^{\circ} \mathrm{C}$, amounting to $34,74 \%$ of that at $24^{\circ} \mathrm{C}$, and even greater than that at 16 and $20^{\circ} \mathrm{C}$. At $12^{\circ} \mathrm{C}$ penetration was not observed in any of the cultivars used. Considering $H$. glycines, the temperatures of $21,3^{\circ} \mathrm{C}$ provided great penetration of $\mathrm{J} 2$ in the susceptible cultivar and at $22,4^{\circ} \mathrm{C}$ in the resistant one. The penetration of $\mathrm{J} 2$ of $H$. glycines at 12 and $32^{\circ} \mathrm{C}$ was similar in both susceptible and resistant cultivars, but corresponded approximately to half of that at the temperatures that were better for penetration.
\end{abstract} resistant cultivar the penetration was not affected $(\mathrm{P} \leq 0,05)$ at

Additional keywords: Root-knot nematode, soybean cyst nematode, parasitism.

\section{RESUMO}

Campos, H.D.; Campos, V.P.; Pozza, E.A. Efeito do tempo, substrato e temperatura na penetração de juvenis do segundo estádio de Meloidogyne javanica e Heterodera glycines em soja. Summa Phytopathologica, v.32, n. 2, p.156-160, 2006.

Observou-se que, independente do substrato, aos 2 dias após a inoculação a penetração de juvenis do segundo estádio (J2) de Meloidogyne javanica nas raízes da soja foi baixa em comparação com os demais períodos. Em areia fina, maior penetração ocorreu aproximadamente aos 4,4 dias após a inoculação. Na mistura de solo + areia grossa, o aumento foi linear no intervalo de 2 a 8 dias após a inoculação. $\mathrm{Na}$ areia fina o número de $\mathrm{J} 2$ de Heterodera glycines no interior das raízes foi mais alto aos 2 dias da inoculação. Na mistura de solo + areia grossa, ocorreram menor número de $\mathrm{J} 2$ de $H$. glycines nas raízes aos 2 dias após a inoculação e tendência de aumento até o 8 o dia. $\mathrm{Na}$ temperatura de $24^{\circ} \mathrm{C}$, ocorreu maior $(\mathrm{P} \leq 0,05)$ penetração de $\mathrm{J} 2$ de $M$. javanica indiferente da resistência da cultivar. Entretanto, na cultivar resistente a penetração não foi alterada $(\mathrm{P} \leq 0,05)$ entre 24 e $28^{\circ} \mathrm{C}$. A $32^{\circ} \mathrm{C}$ ocorreu queda significante no número de $\mathrm{J} 2$ de $M$. javanica nas raízes chegando a $17,97 \%$ daquela em $28^{\circ} \mathrm{C}$ e semelhante àquelas em 16 e $20^{\circ} \mathrm{C}$. Na cultivar suscetível a queda na penetração foi significante aos $28^{\circ} \mathrm{C}$ e $32^{\circ} \mathrm{C}$, da ordem de $34,74 \%$ em relação a $24^{\circ} \mathrm{C}$, porém ainda mais elevada $(\mathrm{P} \leq 0,05)$ do que em 16 e $20^{\circ} \mathrm{C}$. A $12^{\circ} \mathrm{C}$ não ocorreu penetração em qualquer cultivar testada. Para $H$. glycines, a maior penetração na cultura suscetível ocorreu à temperatura de $21,3^{\circ}$ $\mathrm{C}$ e a $22,4^{\circ} \mathrm{C}$ na resistente. A 12 e $32^{\circ} \mathrm{C}$ a penetração de $H$. glycines foi semelhante nas cultivares resistente e suscetível, porém correspondendo a aproximadamente metade daquela observada nas melhores temperaturas.

Palavras-chave adicionais: Nematóide de galhas, nematóide de cisto da soja, parasitismo. 
movimentam-se pelo solo e são atraídos ao local de penetração na raiz $(6,12)$. Nesse momento, sofrem influência de fatores físicos, como tem sido demonstrado para alguns substratos e diferentes tipos de solo. $(13,14,18,23)$.

Na superfície da raiz, a penetração do J2 ainda dependerá da temperatura, da umidade, do local e de suas próprias condições de patogenicidade. Com a penetração do J2, inicia-se o processo de parasitismo e e tem continuidade o desenvolvimento do nematóide. Nesse momento, sofrerá o efeito proporcionado pelo hospedeiro. Herman et al. (9) encontraram maior penetração de $M$. incognita em plantas de soja suscetíveis do que em resistentes aos 8 dias após a inoculação. Entretanto, tem-se encontrado em algumas cultivares resistentes de soja emigração do J2 de $H$. glycines raça 5 após 48 horas da inoculação (8) e questiona-se sobre a ocorrência desse fenômeno também nas suscetíveis. Portanto, são vários os fatores que interferem nesse processo de parasitismo.

Desta forma, objetivou-se, neste trabalho, avaliar o tempo para penetração de $\mathrm{J} 2$ de $M$. javanica e de $H$. glycines raça 3 em soja crescida sobre dois tipos de substratos bem como o efeito de diferentes temperaturas na capacidade de penetração em cultivares suscetíveis e resistentes.

\section{MATERIAL E MÉTODOS}

\section{Obtenção do inóculo de Meloidogyne javanica e de Hete- rodera glycines \\ Meloidogyne javanica foi multiplicado em soja [Glycine} $\max ($ L.) Merrill], cultivar Embrapa 20 (Doko RC), e mantida em vasos em casa de vegetação. Raízes com galhas foram coletadas e os ovos foram extraídos utilizando-se a técnica de Hussey \& Barker (11). A suspensão de ovos livres de impurezas foi colocada em câmara de eclosão e mantida em temperatura de 26 $\pm 2^{\circ} \mathrm{C}$. Os $\mathrm{J} 2$ obtidos durante as primeiras 48 horas foram descartados e utilizados aqueles produzidos com 72 horas de incubação.

O inóculo utilizado de $H$. glycines foi a raça 3 , confirmada através de testes em cultivares e linhagens diferenciadoras, conforme descrito por Niblack (16). Para a sua produção, plantas de soja, cultivar Embrapa 20 (Doko RC), foram inoculadas com ovos e mantidas em vasos de argila em casa-de-vegetação. Trinta dias após, foram retiradas dos vasos e os seus sistemas radiculares colocados sobre peneira de malha de $0,85 \mathrm{~mm}$ sobreposta a outra de $0,18 \mathrm{~mm}$, direcionando jatos de água sobre eles para a remoção das fêmeas e cistos. Para obtenção dos ovos (3), as fêmeas e cistos foram rompidos pressionando um bequer sobre eles na própria peneira de $0,18 \mathrm{~mm}$, acoplada a outra de $0,025 \mathrm{~mm}$. Desta forma, os ovos foram recolhidos na peneira de $0,025 \mathrm{~mm}$ e submetidos a centrifugação em solução de sacarose composta de $454 \mathrm{~g}$ de açúcar por litro de água, a 2000 rpm por 1 minuto, visando-a separá-los das impurezas. Para obter os J2, os ovos foram colocados em câmara de eclosão e mantidos a temperatura de $26 \pm 2^{\circ} \mathrm{C}$. Os $\mathrm{J} 2$ produzidos nas primeiras 48 horas de incubação foram descartados, utilizandose aqueles com 72 horas.

Efeito do tempo e do substrato na penetração dos juvenis J2 Sementes de soja da cultivar Embrapa 20 foram desinfestadas superficialmente com hipoclorito de sódio (1\%) por $1 \mathrm{minu}-$ to e colocadas para germinar em bandejas contendo areia gros- sa umedecida e mantidas em sala climatizada à $27 \pm 2^{\circ} \mathrm{C}$. Três dias após, as sementes germinadas com radícula de $2 \mathrm{~cm}$ foram transferidas para tubos de ensaio contendo $30 \mathrm{~cm}^{3}$ de areia fina quartizífera ou mistura de solo e areia grossa na proporção de 2: 1 , umedecidos, e previamente autoclavada a $120^{\circ} \mathrm{C}$ por $20 \mathrm{minu}-$ tos. Vinte quatro horas após, infestou-se o substrato de cada tubo com $200 \mathrm{~J} 2$ de $M$. javanica ou de $H$. glycines mantendo-os em sala climatizada com temperatura de $27 \pm 2^{\circ} \mathrm{C}$.

Aos 2, 4, 6 e 8 dias após a inoculação, as plântulas foram retiradas jorrando água nas paredes dos tubos. As raízes e a areia contidas nos tubos foram lavadas sobre um conjunto de peneiras de $0,44 \mathrm{~mm}$ sobreposta a de $0,025 \mathrm{~mm}$. O material retido na peneira de $0,025 \mathrm{~mm}$ foi recolhido em solução de sacarose (454g de açúcar por litro de água) em tubos de centrífuga, e centrifugados a $1700 \mathrm{rpm}$ por 1 minuto. O sobrenadante foi vertido em peneira de $0,025 \mathrm{~mm}$, enxaguado e recolhido em câmara de contagem. A seguir, quantificou-se o número de $\mathrm{J} 2$ de $M$. javanica ou de $H$. glycines recuperado em todo o substrato do tubo. Os sistemas radiculares lavados foram submetidos ao clareamento dos tecidos em hipoclorito de sódio a 1,5\% durante 6 minutos, e enxaguados em água corrente. Em seguida, foram transferidos para tubos de vidro onde foi adicionada solução corante diluída até cobrir as raízes. A solução foi preparada com 3,5 gramas de fucsina ácida $+250 \mathrm{~mL}$ de ácido láctico +750 $\mathrm{mL}$ de água destilada (adaptado de Byrd et al., 2) e diluída 1: 29 ( $1 \mathrm{~mL}$ de solução corante inicial $+29 \mathrm{~mL}$ de água destilada). Em seguida, as raízes imersas no corante foram mantidas por 2 minutos em banho-maria com água em ebulição. O resfriamento total foi feito em condições ambiente durante a noite. As raízes foram, então, lavadas para eliminar o excesso de corante e recolocadas nos tubos. Cobriram-se todas as raízes com solução de glicerina 1:1 (glicerina pura + água destilada), as quais foram deixadas em repouso por, no mínimo, duas horas. Em seguida, todas as raízes foram colocadas em lâmina de vidro, adicionando-se sobre elas gotas de glicerina pura. Outra lâmina de vidro foi sobreposta a ela e assim estava pronta para observação ao microscópio de objetiva invertida. Quantificaram-se os números de $\mathrm{J} 2$ de $M$. javanica ou de $H$. glycines dentro de cada segmento radicular, obtendo-se o total por sistema radicular.

Foi empregado o delineamento estatístico inteiramente casualizado, com fatorial 2 x 4, utilizando-se 2 tipos de substrato e 4 épocas de avaliação após a inoculação de cada nematóide, com 6 repetições.

Efeito da temperatura na penetração de juvenis $\mathbf{J 2}$

Plântulas de soja das cultivares Embrapa 20 (suscetível a $M$. javanica e a $H$. glycines), MGBR-46 (resistente a $M$. javanica) e BRSMG Liderança (resistente a $H$. glycines raça 3 ) foram crescidas em areia quartizífera em tubos de ensaio. Vinte quatro horas após o transplantio das sementes germinadas para os tubos, infestou-se o substrato de cada tubo com $100 \mathrm{~J} 2$ de $M$. javanica ou de $H$. glycines. Em seguida, foram transferidos para câmaras climatizadas ou BODs com temperaturas ajustadas para $12,16,20,24,28$ ou $32^{\circ} \mathrm{C}$.

O delineamento empregado foi inteiramente casualizado, em fatorial 2 x 6, com duas cultivares e 6 temperaturas fixas, em 6 repetições, para $M$. javanica ou para $H$. glycines.

Aos 4 dias após a inoculação, quantificaram-se os números de $\mathbf{J} 2$ recuperados na areia e dentro do sistema radicular, seguindo o mesmo procedimento do item anterior. 
A análise de variância, assim como as regressões, foi realizada com o programa SAS version 8 (SAS Intitute Inc., Cary, USA). As fontes de variação significativas pelo teste $\mathrm{F}$ foram empregadas para construir os modelos de regressão. Em seguida, foram plotadas as curvas, e os pontos de máxima foram obtidos pela derivada das equações, a qual foi igualada a zero. Para a fonte de variação sem ajuste de modelos empregou-se o teste de Tukey a $5 \%$ de probabilidade para comparar as médias.

\section{RESULTADOS E DISCUSSÃO}

Efeito do tempo e do substrato na penetração de juvenis J2

Numa análise global, observou-se que, independente do substrato, aos 2 dias após a inoculação, a penetração de J2 de $M$. javanica nas raízes da soja foi baixa em comparação com os demais períodos (Figura 1A). Contudo, aos 4,4 dias após a inoculação, em areia fina e de acordo com o ponto de máximo da equação, houve maior penetração de $\mathrm{J} 2$ por sistema radicular, com redução acentuada após esse período. No substrato solo + areia grossa o aumento foi linear até o $8^{\circ}$ dia após a inoculação (Figura 1A).

$\mathrm{O}$ número de $\mathrm{J} 2$ recuperados no substrato solo + areia grossa foi maior do que na areia fina (Figura 1B). Entretanto, nos dois substratos, houve redução acentuada na recuperação dos J2 entre 2 e 4 dias da inoculação, tendendo a se estabilizar a partir de então até o $8^{\circ}$ dia (Figura $\left.1 \mathrm{~B}\right)$. De modo geral, à medida que a penetração aumentou houve tendência de queda no número de $\mathrm{J} 2$ recuperados no substrato.

$\mathrm{O}$ pequeno número de $\mathrm{J} 2$ de $M$. javanica dentro da raiz da soja aos 2 dias indica, possivelmente, pequena eficiência da propagação do fator atrativo ao $\mathrm{J} 2$ a partir do local de penetra-
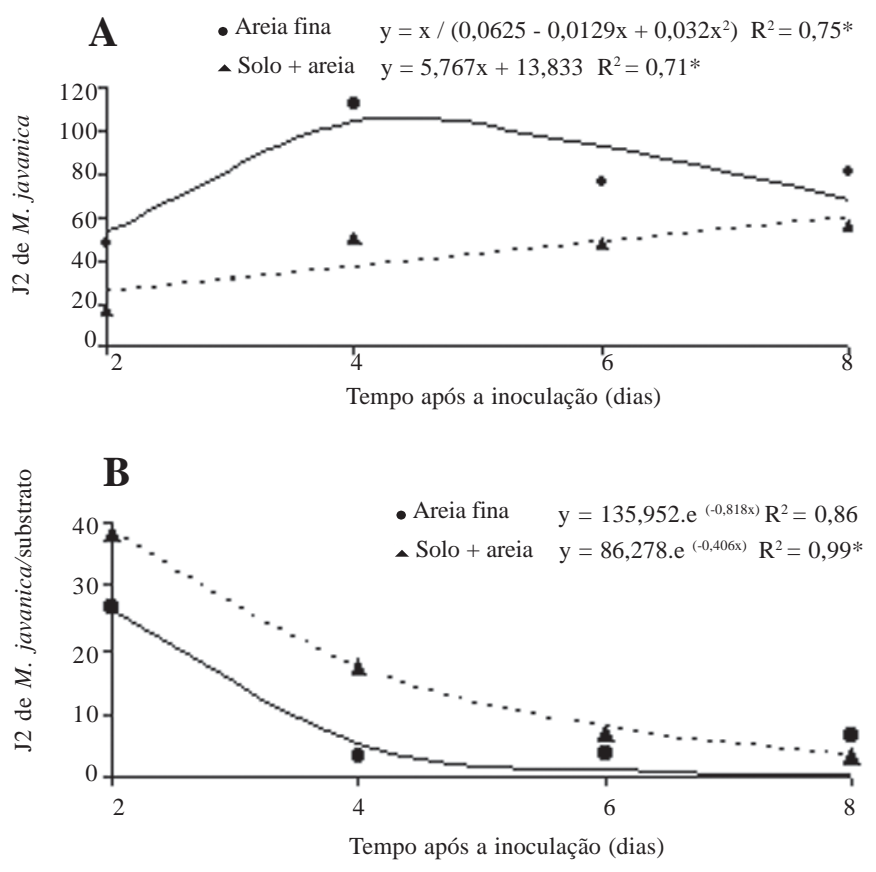

Figura 1. Efeito do tempo após a inoculação na recuperação de juvenis do segundo estádio (J2) de Meloidogyne javanica em raízes de soja suscetível crescidas em substrato de areia fina quartizífera ou em mistura de solo + areia grossa. A) Penetração de J2 nas raízes. B) J2 que ficaram no substrato. ção, aliado a especificidade desse local, pois os $\mathbf{J} 2$ tem a ponta da raiz nova como local preferencial de penetração. Daí muitos pesquisadores terem preferido avaliar a penetração a partir do quarto ou quinto dia da inoculação $(8,10,15,17)$. A queda no número de $\mathrm{J} 2$ dentro da raiz de soja crescida em areia fina após 6 dias comparado com aquele aos 4 não concorreu ao aumento dos J2 no substrato. Provavelmente não houve emigração, mas sim ineficiência no processo de parasitismo (Figura 1A e B). Gourd et al. (8) também trabalhando com $M$. javanica observaram $25 \%$ de penetração em raiz de soja aos 5 dias após a inoculação, entretanto aos dois dias também observaram menor penetração. Para as espécies $M$. incognita, $M$. arenaria e $M$. hapla os autores observaram efeitos semelhantes. Anwar \& Mckenry (1) encontraram menos de $50 \%$ dos $500 \mathrm{~J} 2$ de $M$. arenaria inoculados em mudas de videira suscetível nas raízes aos 4 e 13 dias da inoculação. Herman et al. (10) e Moura et al. (15) encontraram aumento significativo no número de $\mathrm{J} 2$ de $M$. incognita em raiz de cultivar de soja suscetível após 4 dias da inoculação; porém, isto não aconteceu com as cultivares resistentes, que mantiveram baixo número de $\mathrm{J} 2$ na raiz até 16 dias da inoculação devido a alta taxa de emigração para o substrato após 5 dias da inoculação.

Para $H$. glycines, na areia fina, a maior penetração de $\mathrm{J} 2$ nas raízes ocorreu aos 2 dias após a inoculação, decrescendo até os 4 dias (Figura 2A). Ao contrário, na mistura de solo + areia grossa, menor número de $\mathrm{J} 2$ nas raízes foi observado aos 2 dias após a inoculação, com tendência de aumento até o $8^{\circ}$ dia após a inoculação (Figura 2A). O número de $\mathrm{J} 2$ recuperados no substrato areia fina foi semelhante em todos os períodos avaliados (Figura 2B). Entretanto, na mistura de solo + areia grossa houve tendência de redução na recuperação de J2 após 2 dias da ino-
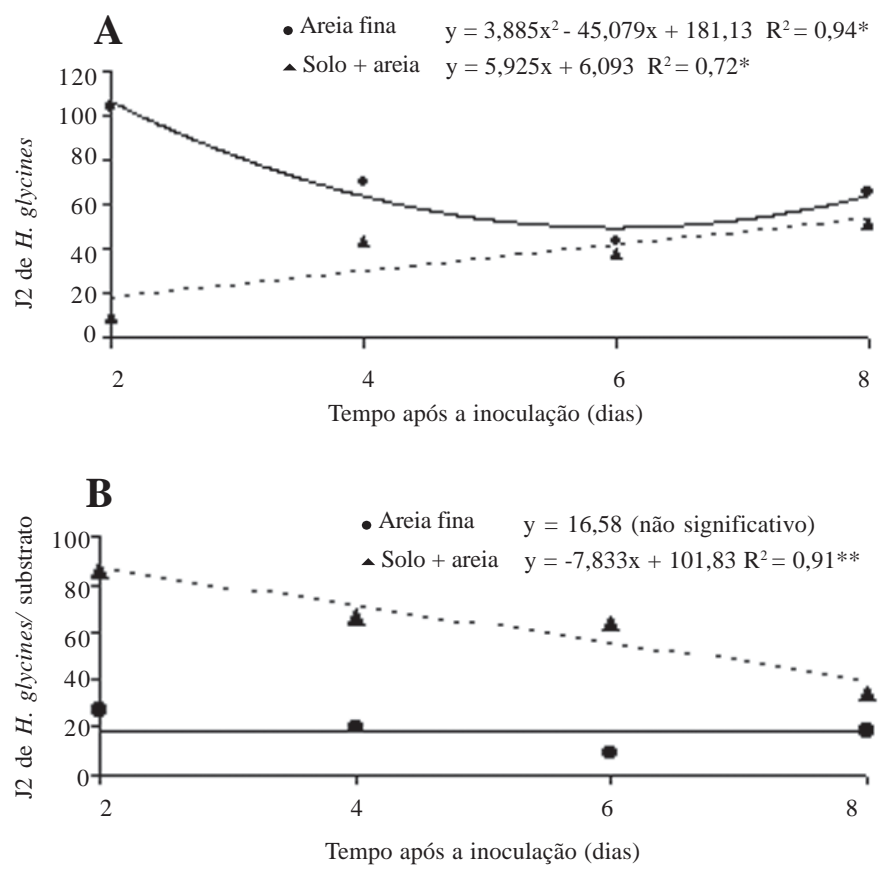

Figura 2. Efeito do tempo após a inoculação na recuperação de juvenis do segundo estádio (J2) de Heterodera glycines em raízes de soja suscetível crescidas em areia fina quartizífera ou em mistura de solo + areia grossa. A) Penetração de J2 nas raízes. B) J2 que ficaram no substrato. 
culação até o 8 o dia. À medida que a penetração aumentou diminuiu o número de J2 no substrato como o ocorrido para $M$. javanica.

Heterodera glycines ao contrário de $M$. javanica foi rapidamente atraído para o local de penetração, devido, talvez, a uma área de atração maior, mais extensa, nas raízes. Todavia, Gourd et al. (8) encontraram maior número de $\mathrm{J} 2$ de $H$. glycines raça 5 nas raízes de soja aos 2 dias após a inoculação, enquanto, para a raça 1 , isso ocorreu aos 10 dias.

De forma geral, com o substrato areia fina quartizífera ocorreu maior penetração de $\mathbf{J} 2$ de $M$. javanica (Figura 1A) ou de $H$. glycines (Figura 2A) nas raízes de soja do que na mistura de solo + areia grossa. Já o número de $\mathrm{J} 2$ recuperados na areia fina quartizífera foi menor em relação a mistura de solo + areia grossa (Figura 1B e 2B). Portanto, a argila proveniente do solo em mistura com areia concorreu para reduzir a penetração dos J2. Prot \& Van Gundy (18) também observaram menor penetração de J2 de $M$. incognita em raízes de tomate quando o solo tinha acima de $22 \%$ de argila e silte e, a partir de $32,7 \%$ não ocorreu penetração. Já em plantas cultivadas em solo com maior concentração de areia, Koenning et al. $(13,14)$ observaram maior migração e penetração de $M$. incognita em algodoeiro e de $H$. glycines em soja.

\section{Influência da temperatura na penetração de juvenis J2}

Embora não tenha ocorrido ajuste a um modelo estatístico, observou-se que na temperatura de $24^{\circ} \mathrm{C}$ ocorreu maior $(\mathrm{P} \leq$ $0,05)$ penetração de $\mathrm{J} 2$ de $M$. javanica independente da resistência da cultivar. Porém, a $32^{\circ} \mathrm{C}$ ocorreu queda significativa chegando a $17,97 \%$ da observada a $28^{\circ} \mathrm{C}$ e semelhante aquelas a 16 e $20^{\circ} \mathrm{C}$ (Figura 3). Tanto na cultivar suscetível quanto na resistente, entre as temperaturas de 12 e $20^{\circ} \mathrm{C}$, não apresentaram variação na quantidade de $\mathrm{J} 2$ penetrados no sistema radicular. A partir de $20^{\circ} \mathrm{C}$ houve aumento significativo na quantidade de $\mathrm{J} 2$ penetrados para a cultivar resistente e a suscetível. As temperaturas que proporcionaram maior penetração foram de $24^{\circ} \mathrm{C}$ e entre 24 e $28^{\circ} \mathrm{C}$ para as cultivares suscetível e resistente, respectivamente, a partir daí, houve queda acentuada (Figura 3). Herman et al. (10) avaliaram a penetração de $M$. incognita em cultivares suscetíveis e resistentes de soja apenas sob temperatura de $25^{\circ} \mathrm{C}$, e também observaram altas taxas de penetração até o quarto dia após a inoculação em ambas as cultivares.

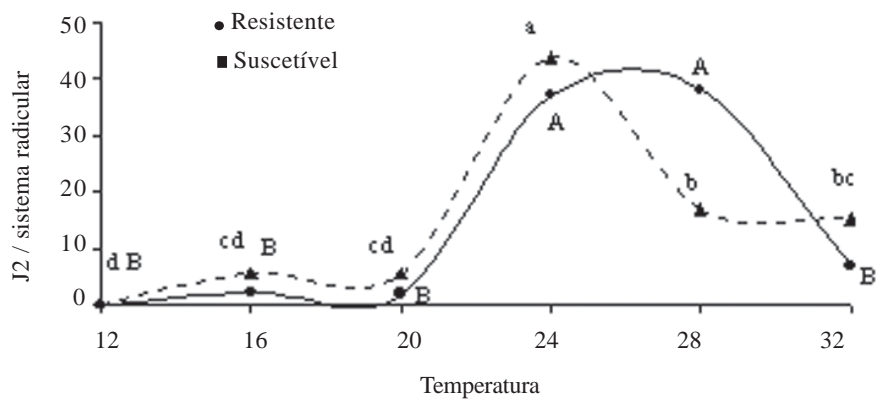

Figura 3. Número de juvenis do segundo estádio (J2) de Meloidogyne javanica em raízes de cultivares de soja resistente (MGBR-46) e suscetível (Embrapa 20) crescidas em diferentes temperaturas, após 4 dias da inoculação. Barras seguidas pela mesma letra não diferem significativamente entre si pelo teste de Tukey a 5\% de probabilidade.
Acima de $24^{\circ} \mathrm{C}$ a temperatura para a penetração dos $\mathrm{J} 2$ nas raízes de soja ainda pode variar entre espécies de Meloidogyne. Gourd et al. (8), trabalhando com as espécies $M$. incognita e $M$. javanica, observaram boa capacidade de penetração em soja suscetível em temperatura variando entre $25^{\circ} \mathrm{C}$ e $31^{\circ} \mathrm{C}$. Resultados semelhantes foram obtidos por Pedrosa et al. (17), quando avaliaram a penetração de $M$. arenaria raças 1 e 2 em genótipos de soja suscetíveis e resistentes nessa faixa de temperatura. No entanto, em raízes de grão-de-bico, Di Vito \& Greco (5) observaram que a penetração de $M$. artiellia não foi satisfatória em temperatura de $30^{\circ} \mathrm{C}$, quando comparada a 15 e $25^{\circ}$ C. De forma general, Taylor \& Sasser (21) e Van Gundy (22) sugeriram a faixa de temperatura entre 25 e $30^{\circ} \mathrm{C}$ como ideal para a penetração de $M$. javanica em seu hospedeiro.

A $12^{\circ} \mathrm{C}$ não ocorreu penetração em qualquer cultivar testada (Figura 3). Considerando que essa temperatura não ocorreu penetração de $\mathrm{J} 2$ de $M$. javanica, a adoção de $10^{\circ} \mathrm{C}$ como temperatura base para calculo de graus-dia, como utilizado por Goodell \& Ferris (7), não parece ser conveniente, provavelmente houve adaptação dessa população a temperaturas mais elevadas no Brasil.

Para H. glycines as temperaturas entre 16 e $28^{\circ} \mathrm{C}$ proporcionaram boa penetração de $\mathrm{J} 2$ independente da resistência ou suscetibilidade da cultivar testada (Figura 4). No entanto, a máxima penetração ocorreu a $21,3^{\circ} \mathrm{C}$ na cultivar suscetível e a $22,5^{\circ} \mathrm{C}$ na cultivar resistente. A redução na penetração a $12 \mathrm{e}$ $32^{\circ} \mathrm{C}$ foi semelhante e também indiferente da reação hospedeiro, porém correspondendo aproximadamente à metade daquela nas temperaturas entre $16 \mathrm{e} 28^{\circ} \mathrm{C}$. Em temperatura de $12^{\circ} \mathrm{Cos} \mathrm{J} 2$ de $H$. glycines penetraram nas raízes das cultivares testadas nas mesmas proporções que em de $32^{\circ} \mathrm{C}$ (Figura 4). Hamblem et al. (9) e Schmitt \& Riggs (19) também observaram que a penetração de $H$. glycines pode ocorrer em temperaturas abaixo de $20^{\circ} \mathrm{C}$ ou acima de $30^{\circ} \mathrm{C}$, porém em baixas proporções. Da mesma forma, Diogo et al. (4) observaram penetração, mas com tendência de redução do número de $\mathrm{J} 2$ dentro das raízes, em temperatura mínima de $17^{\circ} \mathrm{C}$.

A capacidade de penetração de $\mathrm{J} 2$ também pode variar de acordo com a raça do patógeno numa mesma faixa de temperatura. Em temperaturas variando entre 25 a $31^{\circ} \mathrm{C}$, Gourd et al. (8),

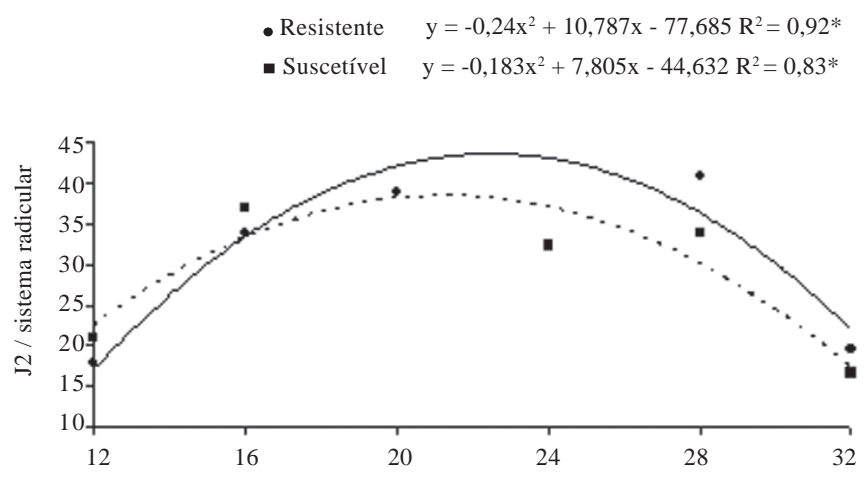

Figura 4. Número de juvenis do segundo estádio (J2) de Heterodera glycines penetrados em raízes de cultivares de soja resistente (BRSMG Liderança) ou suscetível (Embrapa 20), crescidas em diferentes temperaturas, após 4 dias da inoculação. 
verificaram que $44 \%$ dos $\mathrm{J} 2$ da raça 5 de $H$. glycines penetraram em cultivar suscetível contra $24 \%$ dos $\mathrm{J} 2$ da raça 1 .

A reação do hospedeiro caracterizada em resistência e suscetibilidade, não afetou a penetração indicando que o mecanismo que confere resistência é ativado após a penetração. Schmitt \& Riggs (20) também encontraram proporções semelhante de penetração de $H$. glycines raça 3 em cultivares resistentes e suscetíveis de soja.

\section{REFERÊNCIAS BIBLIOGRÁFICAS}

1. Anwar, S.A.; McKenry, M.V. Penetration, development and reproduction of Meloidogyne arenaria on two new resistant Vitis spp. Nematropica, Florida, v.30, n.1, p.9-17, 2000.

2. Byrd, D.W.; Kirkpatrick, T.; Barker, K.R. An improved technique for clearing and staining plant tissues for detection of nematodes. Journal of Nematology, Jay, v.15, n.1, p.142-143, 1983.

3. Dias, W.P.; Silva, J.F.V.; Wain, L.A.; Pereira, J.F. Distribuição de raças de Heterodera glycines no Brasil. In: Sociedade Brasileira de Nematologia (Ed.) O nematóide de cisto da soja: a experiência brasileira. Jaboticabal: Sociedade rasileira de Nematologia, 1999. p.95-103.

4. Diogo, A.M.; Sediyama, T.; Lima, R.D.; Sediyama, C.S. Penetração e reprodução de Heterodera glycines, raça 3, em algumas espécies vegetais. Nematologia Brasileira, Brasília, v.23, n.2, p.24-33, 1999.

5. Di Vitro, M.; Greco, N. Investigation on the biology of Meloidogyne artiellia. Revue de Nématologie, Montrouge Cedex, v.11, n.2, p.223-227, 1988.

6. Endo, B.Y. Pathogenesis of nematode-infected plants. Annual Review Phytopathology, Palo Alto, v.13, p.213- 238, 1975.

7. Goodell, P.B.; Ferris, H. Influence of environmental factors on the hatch and survival of Meloidogyne incognita. Journal of Nematology, Jay, v.21, n.3, p.328-334, 1989.

8. Gourd, T.R.; Schmitt, D.P.; Barker, K.R. Penetration rates by second-stage juveniles of Meloidogyne spp. and Heterodera glycines into soybean roots. Journal of Nematology, Raleigh, v. 25, n. 1, p. 38-41, 1993.

9. Hamblem, M.L.; Slack, D.A.; Riggs, R.D. Temperature effects on penetration and reproduction of soybean-cyst nematode. Phytopathology, St. Paul, v.62, p.762, 1972.

10. Herman, M.; Hussey, R.S.; Boerma, H.R. Penetration and development of Meloidogyne incognita on roots of resistant soybean genotypes. Journal of Nematology, Lake Alfred, v.23, n.2, p.155$161,1991$.
11. Hussey, R.S.; Barker, K.R. A comparison of methods for collecting inocula of Meloidogyne spp., including a new technique. Plant Disease Report, St. Paul, v.57, n.12, p.1025-1128, 1973.

12. Hussey, R.S.; Grundler, F.M.W. Nematode parasitism of plant. In: Perry, R.N.; Wright, D.J. The physiology and biochemistry of free-living and plant-parasitic nematodes. New York: CABI Publishing. 1998. p.213-244.

13. Koenning, S.R.; Anand, S.C.; Wrather, J.A. Effect of within-field variation in soil texture on Heterodera glycines and soybean yield. Journal of Nematology, Jay, v.20, p.373-380, 1988.

14. Koenning, S.R.; Walters, S.A.; Barker, K.R. Impact of soil texture on the reproductive and damage potentials of Rotylenchulus reniformis and Meloidogyne incognita on cotton. Journal of Nematology, Lakeland, v.28, n.4, p.527-536, 1996.

15. Moura, R.M.; Davis, E.L.; Luzzi, B.M.; Boerma, H.R.; Hussey, R.S. Post-infectional development of Meloidogyne incognita on susceptible and resistant soybean genotypes. Nematropica, Florida, v.23, n.1, p.7-13, 1993.

16. Niblack, T.L. The race concept. In: Riggs, E.D.; Wrather, J.A. (Ed.). Biology and management of the soybean cyst nematode. St.Paul: APS, 1992. p.73-86.

17. Pedrosa, E.M.; Hussey, R.S.; Boerma, H.R. Penetration and postinfectional development and reproduction of Meloidogyne arenaria raça 1 and 2 on susceptible and resistant soybean genotypes. Journal of Nematology, Lakeland , v.28, p.343-351, 1996.

18. Prot, J.C.; Van Gundy, S.D. Effect of soil texture and the clay component on migration of Meloidogyne incognita second-stage juvenil. Journal of Nematology, Jay, v.13, n.2, p.213-217, 1981.

19. Schmitt, R.D.; Riggs, R.D. Influence of selected plant species on hatching of eggs and development of juveniles of Heterodera glycines. Journal of Nematology, Lakeland, v.23, n.1, p.1-6, 1991.

20. Schmitt, R.D.; Riggs, R.D. Population dynamic and management of Heterodera glycines. Agricultural Zoology Reviews, Palo Alto, v.3, p.253-269, 1989.

21. Taylor, A.L.; Sasser; J.N. Biology, identification and control of root-knot nematodes (Meloidogyne species). Raleigh: North Carolina State University, 1978. 111p.

22. Van Gundy, S.D. Ecology of Meloidogyne spp.- emphasis on environmental factors affecting survival and pathogenicity. In: Sasser, J.N.; Carter,C.C. An advanced treatise on Meloidogyne. North Carolina: Academic Press, 1985. v.1, p.177-182.

23. Wallace, H.R. Abiotic influences in the soil environment. IN: Zuckerman, B.M.; Mai, W.F.; Rohde, R.A. Plant parantic nematodes. New York: Academic Press, 1971. v.1, p. 257-280. 